\title{
Vertebrate Limb Development: Moving from Classical Morphogen Gradients to an Integrated 4-Dimensional Patterning System
}

\author{
Jean-Denis Bénazet and Rolf Zeller \\ Developmental Genetics, Department of Biomedicine, University of Basel, Mattenstrasse 28, \\ $\mathrm{CH}-4058$ Basel, Switzerland \\ Correspondence: rolf.zeller@unibas.ch
}

A wealth of classical embryological manipulation experiments taking mainly advantage of the chicken limb buds identified the apical ectodermal ridge (AER) and the zone of polarizing activity (ZPA) as the respective ectodermal and mesenchymal key signaling centers coordinating proximodistal (PD) and anteroposterior (AP) limb axis development. These experiments inspired Wolpert's French flag model, which is a classic among morphogen gradient models. Subsequent molecular and genetic analysis in the mouse identified retinoic acid as proximal signal, and fibroblast growth factors (FGFs) and sonic hedgehog $(\mathrm{SHH})$ as the essential instructive signals produced by AER and ZPA, respectively. Recent studies provide good evidence that progenitors are specified early with respect to their PD and AP fates and that morpho-regulatory signaling is also required for subsequent proliferative expansion of the specified progenitor pools. The determination of particular fates seems to occur rather late and depends on additional signals such as bone morphogenetic proteins (BMPs), which indicates that cells integrate signaling inputs over time and space. The coordinate regulation of $\mathrm{PD}$ and $\mathrm{AP}$ axis patterning is controlled by an epithelial-mesenchymal feedback signaling system, in which transcriptional regulation of the BMP antagonist Gremlin1 integrates inputs from the BMP, SHH, and FGF pathways. Vertebrate limb-bud development is controlled by a 4-dimensional (4D) patterning system integrating positive and negative regulatory feedback loops, rather than thresholds set by morphogen gradients.

\section{INTRODUCTION: A HISTORICAL APPRECIATION OF THE PROGRESS ZONE AND MORPHOGEN GRADIENT MODELS}

Eor more than half a century, manipulation F and analysis of vertebrate limb development in different animal models has yielded seminal discoveries that further our knowledge of how growth, specification, and determination are coordinately controlled during embryogenesis. The developing limb bud is a large embryonic field whose cells receive proliferative and positional cues from signals emanating from two instructive signaling centers (organizers). The fore- and hindlimb buds emerge at defined somite positions perpendicular to the primary body axis because of continued growth of the

Editors: James Briscoe, Peter Lawrence, and Jean-Paul Vincent

Additional Perspectives on Generation and Interpretation of Morphogen Gradients available at www.cshperspectives.org

Copyright (C) 2009 Cold Spring Harbor Laboratory Press; all rights reserved; doi: 10.1101/cshperspect.a001339

Cite this article as Cold Spring Harb Perspect Biol 2009;1:a001339 
J.-D. Bénazet and R. Zeller

A

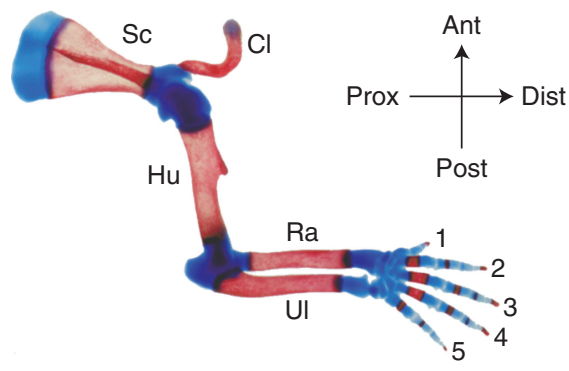

B
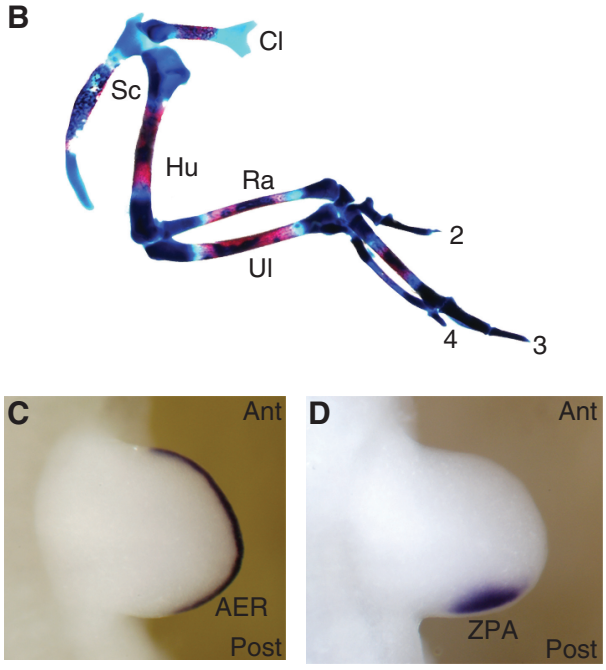

E

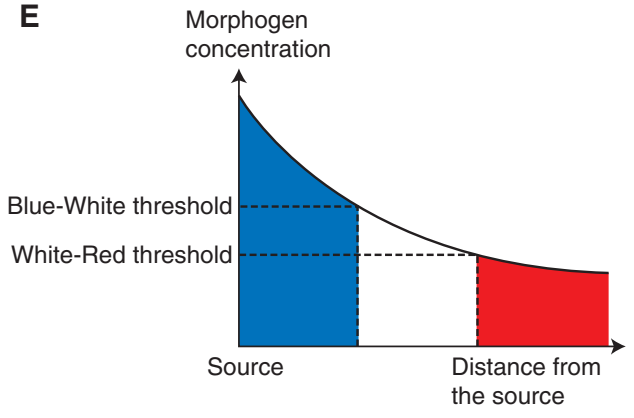

flank mesoderm. The developing limb bud is patterned along three axes: the anteroposterior (AP), dorsoventral (DV), and proximodistal (PD) axes, as is apparent from the morphology of the definitive limb skeleton (Fig. 1A,B). While limb skeletal morphology varies greatly among tetrapods, the underlying basic bauplan is conserved and provides an excellent read-out for normal and altered limb morphogenesis (Shubin et al. 1997). Three main limb skeletal compartments characterize the PD axis: the
Figure 1. Two morpho-regulatory signaling centers control vertebrate limb-bud development. (A) Skeletal preparation of a mouse forelimb at birth. (B) Skeletal preparation of a fetal chicken wing at day 15 of embryonic development. Red and blue histological stains mark ossified bone and cartilage, respectively. Despite morphological differences, the basic bauplan along both axes is conserved. (Proxdist) Proximodistal axis, (ant-post) anteroposterior axis, (Sc) scapula, $(\mathrm{Cl})$ clavicle, $(\mathrm{Hu})$ humerus, $(\mathrm{Ra})$ radius, (Ul) ulna. Digit identities are indicated by numbers. $(C)$ Visualization of the AER by in situ detection of $F g f 8$ transcripts in a mouse limb bud. (D) The ZPA expresses the Shh morphogen. (E) Wolpert's French flag model: A concentration gradient forms by diffusion of a morphogen from a source and positional information is determined in groups of cells by inducing distinct responses to specific concentration thresholds (indicated by blue, white, and red).

proximal stylopod, followed by the zeugopod and the distal autopod (Fig. 1A,B). The AP limb axis is congruent with the primary body axis and manifests itself best in the skeletal morphology of the zeugopod (radius/ulna and tibia/fibula) and in the distinct identities of the digits bearing autopod (Fig. 1A,B). Five distinct digits form in mice and humans with digit 1 (thumb) having the most anterior and digit 5 (little finger) the most posterior identities. AP digit identities are morphologically defined by 
their position and the number, length, and shapes of the metacarpal bones and the phalanges (Fig. 1A). It is generally accepted that the identities of the limb skeletal elements reflect the establishment of positional identities during limb-bud development. Although this article focuses on the role of the graded signaling interactions that control establishment of the PD and AP limb-bud axes, during outgrowth, the limb bud is also polarized along its DV axis from early developmental stages onwards (for review, see Zeller and Duboule 1997; Niswander 2003).

The AER Controls Outgrowth and Patterning of the PD Limb-bud Axis

The emerging limb bud consists of an ectodermal pocket initially "filled" with apparently naïve and undetermined mesenchymal cells that derive from the embryonic flank mesoderm. During the onset of outgrowth, a morphologically distinct ectodermal thickening that consists of a partially stratified epithelium forms at the distal tip, which is called the apical ectodermal ridge (AER) (Fig. 1C). 60 years ago, Saunders performed a series of microsurgical experiments in chicken limb buds, establishing that removal of the AER causes a developmental arrest, which truncates the chicken wing skeleton (Saunders 1948). Removing the AER at progressively earlier stages results in truncations of the limb skeleton at progressively more proximal levels. From these experiments, Saunders concluded that the AER is required for outgrowth and patterning of the PD limb axis. In particular, it seemed that the time mesodermal cells spend under the influence of the AER is relevant with respect to their subsequent PD identities, such that the distal-most cells depend on the AER for the longest time. Furthermore, grafts of an AER to a recipient limb bud induces ectopic PD outgrowth, revealing its strong growth-promoting potential, while its removal results in cell death (Fallon et al. 1983). These and other studies led Summerbell and Wolpert to formulate the so-called progress zone model (Fig. 2A) (Summerbell et al. 1973). This model, which has only recently been challenged and modified as a consequence of extensive molecular analysis (see later) predicts that acquisition of PD identities depends on the time spent by proliferating undetermined cells in the distal mesenchyme (progress zone) under the influence of AER signals. As the progress zone is displaced distally, the more proximal cells are no longer under the influence of the AER, which causes determination of their positional identities. Mesenchymal cells "left behind" early acquire more proximal identities, whereas progenitor cells staying under influence of the AER longer acquire progressively more distal identities (Fig. 2A). The progress zone model introduced for the first time the notion of time as an important component of morphogenetic signaling. In 1993, Niswander and colleagues identified fibroblast growth factors (FGFs) as the relevant signals produced by the AER to induce PD limb axis formation. In particular, $\mathrm{PD}$ outgrowth is rescued by exogenous application of FGFs on AER removal and FGFs are normally expressed by the AER (Fig. 1C) (Niswander et al. 1993). This study provided the first molecular insights into how AER-FGF signaling controls in PD outgrowth and patterning.

\section{The ZPA is an Organizer that Controls AP Limb-bud Patterning}

About 50 years ago, Zwilling (1956) was trying to understand how the AER is maintained and in the course of these studies he identified a region within the posterior mesenchyme with the property to produce an AER maintenance factor. A good decade later, Saunders and Gasseling (1968) discovered that transplantation of this posterior mesenchyme to the anterior margin of recipient chicken wing buds results in induction of mirror image duplications of all digits, while grafts to more posterior positions result in progressively less complete duplications (Tickle et al. 1975). This respecification activity was termed "polarizing activity" and the posterior region was called the zone of polarizing activity (ZPA) or polarizing region (Fig. 1D) because of its 
J.-D. Bénazet and R. Zeller
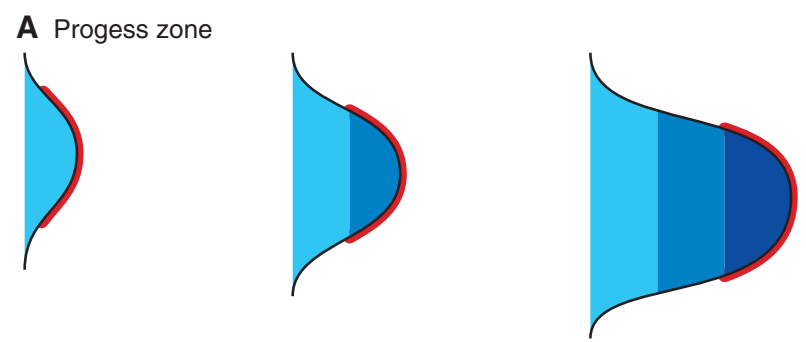

AER-FGF

Progenitor domains:

Stylopod
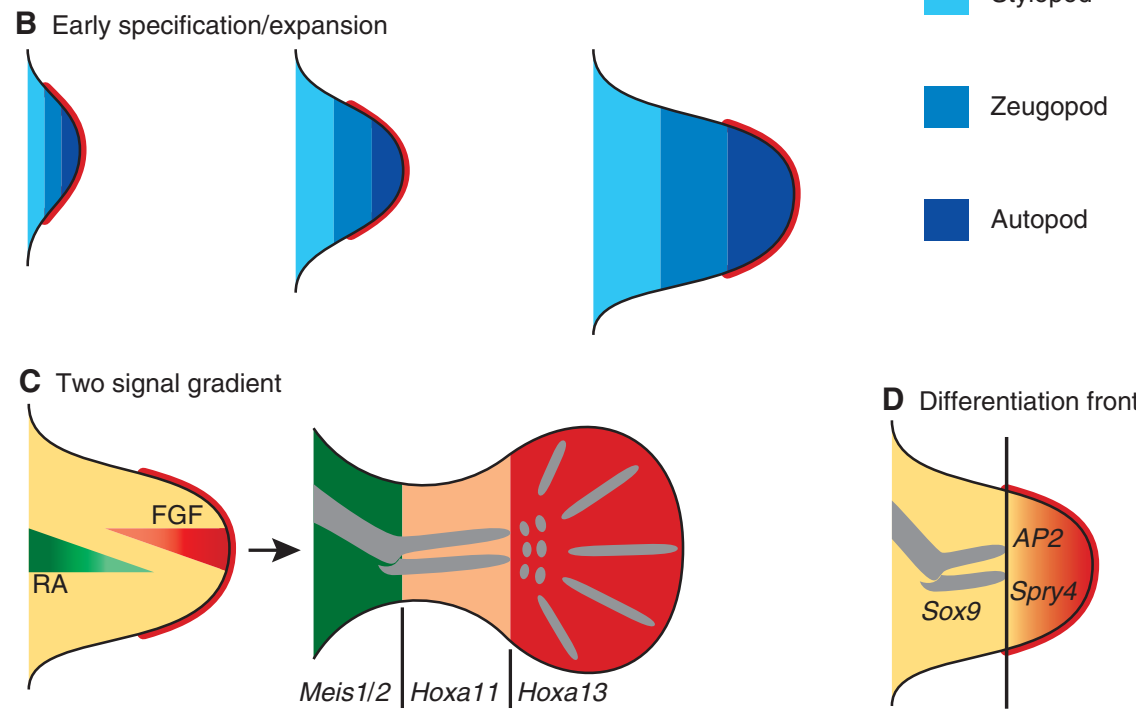

Figure 2. Models and mechanisms of PD limb axis morphogenesis. (A) The original progress zone model. PD positional information values depend on the time cells have spent in the progress zone under the influence of the AER. Stylopod identity is acquired early, whereas zeugopod and autopod identities are specified at progressively later time points. The sequence of skeletal elements is specified from proximal to distal. (B) Early specification/ expansion model. PD positional information is specified very early during initiation of limb-bud development and the specified territories expand sequentially during distal progression of limb-bud outgrowth. $(C)$ Two signal gradient model. Cells are specified by a proximal to distal RA gradient emanating from the embryonic flank/proximal limb bud and by a distal to proximal gradient of AER-FGF signaling. Integration of these two signals over space and time provides the cells with their positional values. The Meis1/2, Hoxa11, and Hoxa13 expression domains mark the three PD territories. $(D)$ The differentiation front model. AER-FGF signaling keeps the distal mesenchyme in an undifferentiated state. Sprouty4 (Spry4) and AP2 are molecular markers of this undifferentiated zone, while Sox 9 marks differentiating chondrocytes. The differentiation front separates these two domains and is displaced distally during progression of limb-bud outgrowth.

properties of a classical embryonic organizer. Wolpert (1969) proposed that the ZPA specifies positional information in the limb-bud mesenchyme by secreting a diffusible molecule that forms a posterior (high) to anterior (low) gradient. Wolpert's model became famous as the "French Flag model" as it proposes that mesenchymal cells receive their positional identities by responding to specific thresholds of the morphogen gradient (Fig. 1E). In contrast to other proposals (see e.g., French et al. 1976), Wolpert's morphogen hypothesis was able to explain the different patterns of digit duplications observed by grafting ZPAs into various positions in recipient limb buds. In support, Tickle (1981) established that the numbers and identities of duplicated digits depend on the number of ZPA cells grafted. 
A small number of ZPA cells $(\sim 30)$ induce digits with anterior identities, whereas specification of additional posterior digits requires many more cells $(\geq 150)$. With the dawn of molecular biology, the hunt for Wolpert's morphogen was initiated and retinoic acid (RA) was first identified as a diffusible molecule that is able to induce digit duplications comparable to ZPA grafts (Tickle et al. 1982; Summerbell and Harvey 1983). Despite initial claims (Thaller and Eichele 1987), it was never shown that endogenous RA is produced or secreted by ZPA cells to form a posterior to anterior gradient, which together with other experimental results excluded it from being the morphogen produced by the ZPA (Wanek et al. 1991). In 1993, Tabin and coworkers succeeded in identifying sonic hedgehog $(\mathrm{SHH})$ as the true morpho-regulatory signal peptide produced by the ZPA (Fig. 1D) (Riddle et al. 1993).

\section{RA AND FGFS: EVIDENCE THAT TWO OPPOSING SIGNALING GRADIENTS CONTROL PD LIMB AXIS SPECIFICATION}

RA Acts as a Proximalizing

Morphogenetic Signal

Although a direct role for RA in specifying the AP axis has been excluded, experimental manipulation of chicken limb buds and genetic analysis in the mouse show that RA functions in specification of the PD axis for which there are now good molecular markers. For example, the expression domains of the Meis 1/2 transcription factors mark the proximal limb-bud mesenchyme corresponding roughly to the future stylopod, whereas Hoxa11 and Hoxa13 mark the prospective zeugopod and autopod territories (Fig. 2C) (for review, see Tabin and Wolpert 2007). Their expression is altered by ectopic RA signaling such that the expression of Meis $1 / 2$ expands distally on RA treatment (Mercader et al. 2000) or genetic inactivation of CYP26B1, an enzyme involved in the degradation of RA (Yashiro et al. 2004). Concurrently, the distal expression of Hox genes is reduced, revealing that exogenous RA proximalizes the limb-bud mesenchyme (Mercader et al. 2000). RA is synthesized by retinaldehyde dehydrogenases (RALDH) and in particular, RALDH2 is expressed in the limb-bud flank mesenchyme during onset of outgrowth. In the mouse, inactivation of the Raldh2 gene arrests embryogenesis and disrupts initiation of limbbud development (Niederreither et al. 1999; Niederreither et al. 2002). The latter is rescued by providing exogenous RA during the onset of limb-bud development (Niederreither et al. 2002). Taken together, these studies indicate that RA is synthesized in the proximal mesenchyme and spreads into the distal limb bud, in which it is actively degraded (Yashiro et al. 2004). This is presumed to result in a PD gradient of RA activity, whereby high levels of RA would specify proximal cell fates and inhibit distal ones (Fig. 2C).

\section{AER-FGF Signaling Promotes Distal Progression of Limb-bud Morphogenesis}

The seminal discovery that FGFs can replace the AER and restore distal outgrowth on AER removal in chicken limb buds (Niswander et al. 1993) resulted in the systematic genetic analysis of the essential FGF signaling function during mouse limb-bud development. Fgf10 is expressed by the limb-bud mesenchyme and is essential to establish AER-FGF signaling, which is in turn required to maintain Fgf 10 expression (Ohuchi et al. 1997; Sun et al. 2002). Indeed, development of Fgflo-deficient limb buds is arrested at a very early stage, resulting in agenesis of limbs (Min et al. 1998; Sekine et al. 1999). Four Fgf ligands (Fgf4,-8,-9,-17) are expressed by the AER (for review, see Martin 1998) and extensive genetic analysis has provided insight into their overlapping, respectively redundant functions during outgrowth and PD patterning of mouse limb buds. Rather unexpectedly, concurrent inactivation of all three $\mathrm{Fgfs}$ expressed predominantly by the posterior AER ( Fgf4,-9,-17) does not alter limb-bud development (Mariani et al. 2008). In contrast, loss of $F g f 8$, which is the first and only Fgf ligand expressed by the entire AER from early stages onwards (Fig. 1C), disrupts 
formation of the proximal-most limb skeletal element, the stylopod (Lewandoski et al. 2000; Moon and Capecchi 2000). This unexpected early and transient disruption of PD outgrowth is rescued by precocious and uniform activation of Fgf4 in the Fgf8-deficient AER, which enables almost normal development of the more distal limb skeleton (Sun et al. 2002; Lu et al. 2006). Indeed, concurrent inactivation of both $\mathrm{Fgf8}$ and Fgf4 causes a complete arrest of limb-bud development and limb agenesis (Sun et al. 2002; Boulet et al. 2004). These genetic studies also revealed that transient expression of Fgf8 and Fgf4 during initiation of limb-bud outgrowth is sufficient for specification of the entire PD axis, but the progressive proliferative expansion of such specified limb segments is disrupted (Sun et al. 2002). A recent genetic study shows that the other AER-FGFs, in particular FGF9, contribute to this proliferative expansion of the early specified PD axis such that higher AER-FGF levels are required for formation of more distal limb skeletal structures (Fig. 2B,C) (Mariani et al. 2008). Taken together, this genetic analysis reveals an instructive role of AER-FGF signaling in the specification and proliferative expansion of the PD limb-bud axis.

\section{Early Specification and Progressive Expansion/Differentiation Front Models}

These results, in particular the loss of proximal but not distal skeletal elements in Fgf8-deficient mouse limb buds (Lewandoski et al. 2000), were difficult to reconcile with the classical progress zone model (Fig. 2A). Furthermore, fatemapping studies in chicken limb buds provided good additional evidence that progenitor pools with distinct PD identities are specified very early and then expanded sequentially by proliferation (Dudley et al. 2002). Removal of the AER at progressively later stages simply eliminates the distal mesenchyme containing the specified but not yet expanded progenitor pools. Taken together, these studies provide a straightforward alternative explanation for the loss of distal skeletal elements following
AER extirpation (Dudley et al. 2002). These and other results led to the proposal of the early specification/expansion model as a valid alternative to the progress zone model (Fig. 2B) (Dudley et al. 2002; Sun et al. 2002). According to this model, AER-FGF signaling controls survival and sequential proliferative expansion of PD territories in a dose- and timedependent fashion. Although, no molecular markers for the proposed early specification phase are known, it has been shown that this early specification indeed occurs and endows mesenchymal cells with the potential to sort themselves out according to their PD identities (Barna and Niswander 2007). Furthermore, these studies corroborate the proposal by Mercader et al. (2000), which states that PD limb-bud identities are specified by two early, opposing signals, namely RA and AER-FGFs as proximalizing and distalizing signals, respectively (Fig. 2C). PD positional identities are likely specified as a consequence of cells integrating these signaling cues. During the onset of limb-bud development, the source of RA and AER-FGFs are very close, but their distance increases with outgrowth of the limb bud, such that proximal cells are exposed to RA for much longer than AER-FGFs, whereas the reverse applies to distal cells. Similar to specification of AP positional identities (see the following), the integration of the dose and time of exposure to both signals likely provides cells with their PD positional cues (Fig. 2C). In an attempt to integrate the molecular and genetic knowledge with the classical experimental studies, Tabin and Wolpert (2007) proposed a modified model that better approximates all known experimental facts (Fig. 2D). This model states that during the proliferative expansion of the PD axis, distal mesenchymal cells maintain an undifferentiated state because of exposure to AER-FGFs. As the PD limb axis expands distally, proximal mesenchymal cells are no longer under the influence of AERFGFs, which results in the determination of their PD fates and initiation of differentiation. Therefore, the proximal limit of cells receiving AER-FGF signals at a given development time point defines a "differentiation front." This 
differentiation front prefigures the PD sequence by which the chondrogenic elements of the limb skeleton become apparent during subsequent mesenchymal condensation of the cartilage models (Fig. 2D).

\section{OF SPACE AND TIME: HOW THE SHH MORPHOGEN SPECIFIES THE AP LIMB AXIS AND DIGIT IDENTITIES}

\section{Setting Up the ZPA and Morphogenetic SHH Signaling}

As described before, the ZPA is located in the posterior limb-bud mesenchyme and specifies AP identities in the mesenchyme by morphogenetic SHH signaling (Fig. 1D). Mapping of polarizing activity by grafting experiments in chicken embryos established that diffuse polarizing activity is already present in the presumptive limb field (Hornbruch and Wolpert 1991; Tanaka et al. 2000). During subsequent activation of Shh expression and initiation of limb-bud outgrowth, polarizing activity is posteriorly restricted and vastly up-regulated. While genetic evidence in the mouse implicates both RA and FGF8 in Shh activation (Lewandoski et al. 2000; Niederreither et al. 2002), these two signals are unlikely to restrict polarizing activity posteriorly. During emergence of the limb bud, the expression of the 5 'most members of the Hoxd gene cluster is activated within the posterior limb-bud mesenchyme. Genetic analysis has shown that the 5'Hoxd transcriptional regulators are essential for activation of Shh expression in the posterior limb-bud mesenchyme (Fig. 3A) (Tarchini et al. 2006). Indeed, cell biochemical studies have revealed a direct interaction of Hoxd proteins with the cis-regulatory limb-bud enhancer region of the Shh gene (Capellini et al. 2006). In addition, the dynamic expression of the Hand 2 transcription factor in the limb field mesenchyme and its posterior restriction during onset of limb-bud development parallels the posterior restriction of polarizing activity. Genetic studies in mouse and zebrafish embryos have indeed implicated HAND2 in the activation of Shh expression in both limb and fin buds (for review, see Cohn 2000). Additional genetic studies in the mouse revealed a mutual antagonistic interaction of HAND2 with GLI3, which suggest a potential mechanism by which these two transcriptional regulators prepattern the limb field before activation of Shh expression (Fig. 3A) (Ros et al. 1996; te Welscher et al. 2002a). This prepatterning mechanism may not only restrict Shh activation to the posterior mesenchyme, but also specify anterior and posterior limb-bud compartments already during the onset of limb-bud development (Fig. 3A).

$\mathrm{SHH}$ is one of the three vertebrate homologs of the segment polarity gene Hedgehog and its genetic analysis has revealed essential functions in a large number of morpho-regulatory processes (for review, see Varjosalo and Taipale 2008). During limb-bud development, Shh expression domain marks the ZPA and naïve fibroblasts engineered to express $\mathrm{SHH}$ are endowed with polarizing activity as grafts induce complete mirror image duplications (Riddle et al. 1995). Genetic inactivation of Shh disrupts establishment of the AP limb axis as the zeugopod is reduced to one anterior bone, the radius, and the autopod is lost with exception of one rudimentary digit of likely anterior character (Fig. 3B) (Chiang et al. 2001; Kraus et al. 2001).

The active SHH signaling peptide is generated by autoproteolytic cleavage of the fulllength protein and is covalently modified by the addition of cholesterol and palmitate moieties (Mann and Beachy 2004). This modified peptide forms a posterior to anterior gradient in the developing limb bud (Fig. 3C) (Zeng et al. 2001; Li et al. 2006). Genetic alteration such that the active $\mathrm{SHH}$ peptide is no longer cholesterated increases its spread, which results in formation of additional anterior digits (preaxial polydactyly) (Li et al. 2006). In contrast, the palmitoyl modification increases long-range signaling by promoting the formation of multimeric SHH ligand complexes (for more details, see Chen et al. 2004). Limb buds of mouse embryos deficient for the enzyme for palmitoylation lack digit 2 and 
J.-D. Bénazet and R. Zeller

A Prepatterning

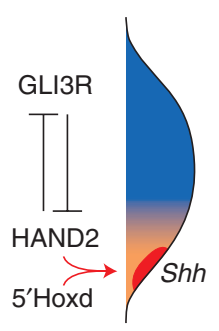

C Spatial gradient

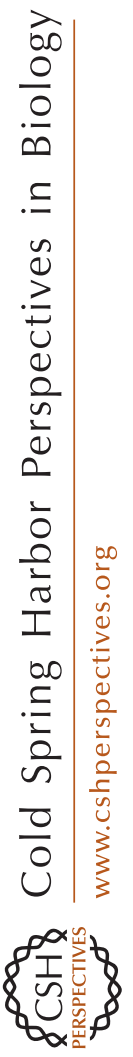

SHH function

Early
B Shh loss of function

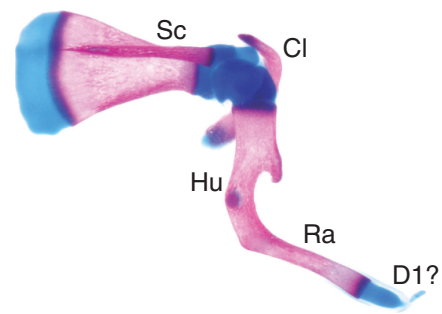

D Temporal gradient

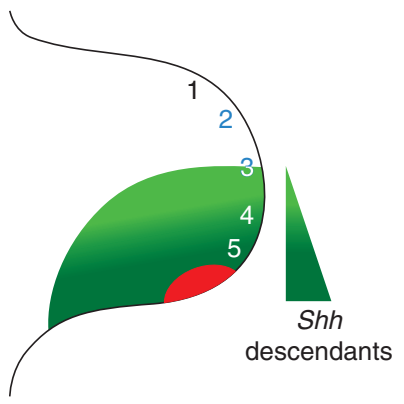

E Specification preceeds expansion and determination

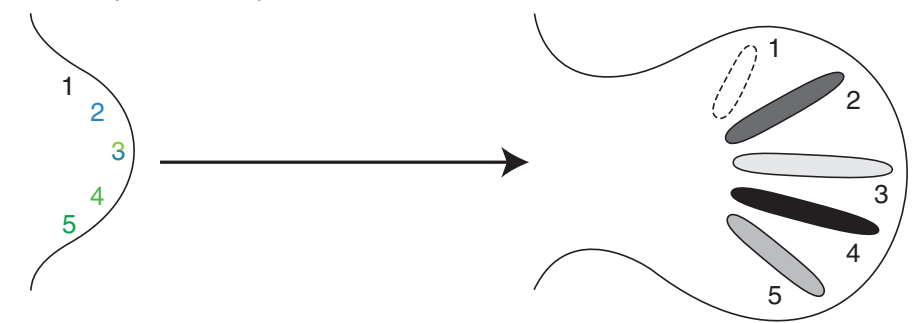

specification (12h) Expansion and determination (4, 2, 5 and 3)

Figure 3. Models and mechanisms for SHH-mediated AP limb axis patterning $(A)$ The early limb bud is already prepatterned by an antagonistic interaction between HAND2 (orange) and the repressor form of GLI3 (GLI3R, dark blue) transcription factors. Nested expression of $5^{\prime}$ Hoxd genes and HAND2 participate in activation of Shh expression. (B) Skeletal preparation of a Shh deficient mouse limb at birth. (C) Spatial gradient model. Diffusion of the SHH peptide secreted by the ZPA generates a GLI3R gradient across the limb bud (graded blue) by inhibiting processing of full-length GLI3. The red line indicates the threshold values predicted by Wolpert's French flag model (Fig. 1E). (D) Temporal gradient model. Descendants of Shh expressing ZPA cells contribute to the progenitor domains of digit 3 to 5. Cells having expressed Shh for a short time contribute to digit 3, whereas the progenitor domains of digits 4 and 5 contain cells having expressed Shh for progressively longer times. Progenitors forming digit 2 and parts of digit 3 are specified by long-range $\mathrm{SHH}$ signaling. (E) Genetic analysis of the temporal requirement of $\mathrm{SHH}$ in the mouse shows digit identities are specified early. Subsequently, $\mathrm{SHH}$ is required for proliferative expansion of progenitor pools and determination of specified identities. Determination of digit identities in the mouse occurs in the following sequence: digit 4 (first), 2, 5, and 3 (last). 
display fusions of digits 3 and 4 , which is indicative of reduced long-range signaling (Chen et al. 2004).

Cellular Response to Morphogenetic SHH Signaling

Cells responding to $\mathrm{SHH}$ will transduce the signal to the nucleus via activation of the GLI1/2 transcriptional activators and inhibition of the GLI3 transcriptional repressor (GLI3R). Genetic analysis shows that Gli1 and Gli2 are not essential for limb-bud development (for more details, see Ahn and Joyner 2004 and references therein). In contrast, inactivation of Gli3 results in the formation of several additional digits with no AP identities, which reveals the essential role of GLI3 in specifying the number and identity of digits (for review, see Theil et al. 1999). A polydactylous autopod also forms when both Shh and Gli3 are inactivated together, which indicates that one of the main functions of $\mathrm{SHH}$ is to counteract GLI3R (Litingtung et al. 2002; te Welscher et al. 2002b). In absence of SHH signaling, the full-length GLI3 protein is constitutively processed to GLI3R, while this process is inhibited by $\mathrm{SHH}$ signaling (for review, see Varjosalo and Taipule 2008). Indeed, evidence for the existence of an intracellular, anterior (high) to posterior (low) GLI3R gradient opposing $\mathrm{SHH}$ has been obtained in the limb-bud mesenchyme (Fig. 3C) (Wang et al. 2000). This GLI3R repressor gradient seems required to establish the polarized expression of genes involved in AP patterning such as, for example, 5'Hoxd genes (Zúñiga and Zeller 1999; Litingtung et al. 2002; te Welscher et al. 2002b).

The notion of opposing spatial gradients of $\mathrm{SHH}$ and GLI3R (Fig. 3C) is complicated by the fact that the descendants of Shhexpressing cells give rise to the posterior half of the limb bud, namely digits 3 to 5 as revealed by genetic cell lineage marking experiments (Fig. 3D; compare to Fig. 3C) (Harfe et al. 2004). Descendants born early, i.e., derived from ZPA cells having expressed Shh for only a short time, will be incorporated into digit 3 together with anterior cells having responded to long-range $\mathrm{SHH}$ signaling. Descendants of cells having expressed Shh for longer times will be incorporated into the posterior digits 4 and 5 (Fig. 3D) (Harfe et al. 2004). In contrast, the anterior digit 2 is specified in response to long-range $\mathrm{SHH}$ signaling (Fig. 3C) (Ahn and Joyner 2004). In agreement, limiting morphogenetic $\mathrm{SHH}$ signaling to a short time window is sufficient to pattern anterior, but not posterior digits (Scherz et al. 2007). In contrast, decreasing overall $\mathrm{SHH}$ activity without altering the time of signaling is sufficient to specify posterior digits, but the proliferative expansion of the autopod territory is affected. These studies indicate that the time spent expressing Shh provides cells with a kinetic memory relevant to specification of their AP identities (Fig. 3D) (Harfe et al. 2004; Scherz et al. 2007; for review, see Zeller 2004). However, mesenchymal cells not only integrate their response to $\mathrm{SHH}$, but they also modulate their responsiveness (Ahn and Joyner 2004; Scherz et al. 2007). Posterior cells are exposed to much higher levels of $\mathrm{SHH}$ for longer times than anterior cells, which results in them being desensitized in comparison to anterior cells. These studies reveal the complexity by which the response to $\mathrm{SHH}$ signaling is integrated over time and space in an expanding system such as the autopod territory.

\section{BACK TO THE FUTURE: HOW AND WHEN ARE DIGITS SPECIFIED AND/OR DETERMINED?}

Wolpert's French flag model (Fig. 1E) provided a simple conceptual framework for understanding how and when digit identities are specified/ determined by the limb patterning system. This question has been revisited recently in several studies. Cyclopamine-mediated blocking of SHH signal transduction shortly after its initiation inhibits proliferation and patterning of the posterior-most digits as a consequence of shortening the exposure to $\mathrm{SHH}$ signaling (Towers et al. 2008), which agrees with the temporal gradient model (Fig. 3D). If proliferation of the digit progenitor pool is, however, blocked by a cell-cycle inhibitor, then 
the expansion of the presumptive digit territory is inhibited. As a consequence, only digits with posterior identities form because of exposure of the remaining small autopod territory to high levels of SHH signaling (Towers et al. 2008). In addition, genetic inactivation of $S h$ from defined developmental time points onwards induces digit losses in a sequence not consistent with a morphogen gradient type patterning mechanism (Zhu et al. 2008). Rather, the sequence of digit loss reflects the sequence by which the digits normally condense. This study indicates that $\mathrm{SHH}$-mediated specification of digit identities occurs within the first 12 hours of $\mathrm{SHH}$ signaling and that $\mathrm{SHH}$ activity is continuously required to generate the required number of cartilage progenitor cells (Fig. 3E) (Zhu et al. 2008).

Furthermore, evidence is accumulating that digit identities are fixed (determined) much after specification and expansion of
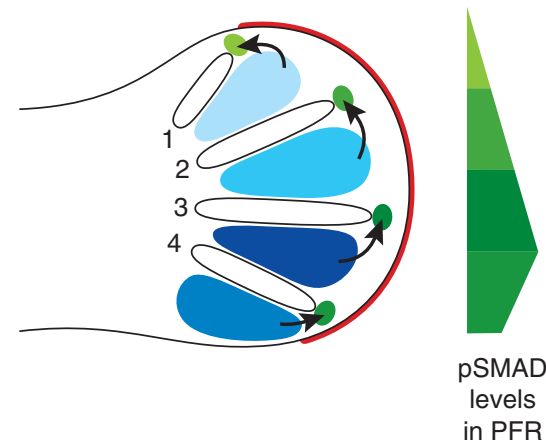

Figure 4. The role of BMP signaling from the interdigital mesenchyme in determination of digit identities. Graded BMP signaling from the interdigital (ID) mesenchyme (blue) in the chicken foot primordia is involved in determining the identities of digits 1 to 4 at late developmental stages. The distal phalanx of individual digits form from the sub-AER mesenchyme, which is therefore called phalanx forming region (PFR). The activity of phosphorylated SMAD ( $\mathrm{pSMAD}$ ) proteins, which are the intracellular mediators of BMP signal transduction, is graded within the PFR (green), such that each digit has its characteristic pSMAD activity signature. Note that the PSMAD activity in the PFR of the posterior-most digit 4 is lower than the one of digit 3 . AER is indicated in red. their progenitor domains. In chicken limb buds, this late determination process is governed by instructive BMP signaling from the interdigital (ID) mesenchyme before its elimination by cell death (blue shaded regions in Fig. 4). This became evident as a consequence of the serendipitous discovery that digit identities can still be altered at late developmental stages by manipulating BMP signaling in the footplate of chicken hindlimb buds (Dahn and Fallon 2000). The targets of this instructive BMP signaling from the ID mesenchyme are the cells located at the distal tip of the forming digit models. This region of mesenchyme located directly under the AER is called the phalanx-forming region (PFR) (Suzuki et al. 2008). Suzuki and coworkers established that PFR of a particular digit is characterized by its unique BMP activity signature (green shaded areas in Fig. 4). The activities of SMAD proteins are higher in posterior than anterior PFRs, with exception of the most posterior digit 4 (Fig. 4). In addition, FGF signaling from the AER also participates in this late determinative process by regulating the number of phalanges formed, which is a defining hallmark of digit identities (Sanz-Ezquerro and Tickle 2003). At present, it is not known which BMP ligands generate these activity signatures and how BMP signaling is integrated with AER-FGF signaling.

\section{INTEGRATION OF PD AND AP PATTERNING BY INTERACTING SIGNALING SYSTEMS WITH SELF-REGULATORY PROPERTIES}

Tickle (1981) already observed that polarizing grafts are most potent when placed in direct contact with the AER. Subsequently, it was discovered that maintenance and propagation of Shh expression requires AER-FGF signaling as part of a positive epithelial-mesenchymal (E-M) feedback loop operating between the ZPA and the AER (Laufer et al. 1994; Niswander et al. 1994). The BMP antagonist Gremlin1 (GREM1) was identified as a crucial mesenchymal component in this E-M feedback signaling system (Zúñiga et al. 1999; Khokha et al. 2003; Michos et al. 2004). GREM1 is 


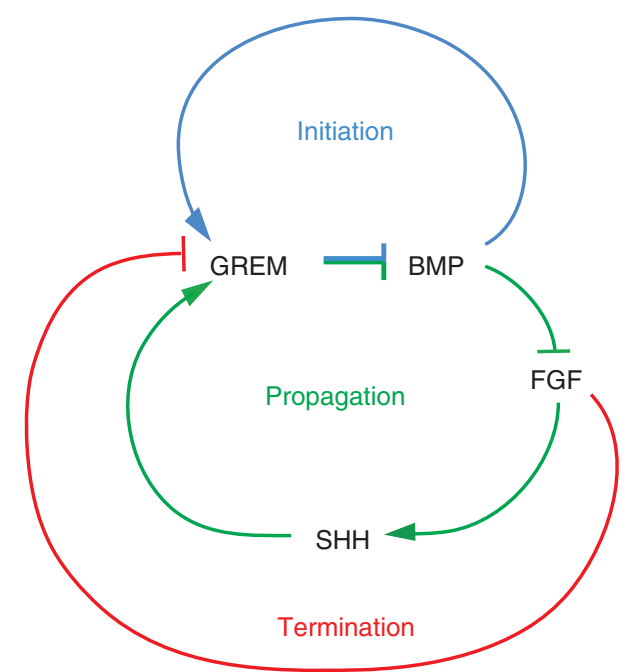

Figure 5. Interlinked signaling feedback loops control initiation, propagation, and termination of $\mathrm{E}-\mathrm{M}$ feedback signaling. The SHH/GREM1/FGF E-M feedback loop is required for maintaining and propagating $\mathrm{SHH}$ signaling by the ZPA and up-regulation of FGF signaling in the AER. In mouse limb buds lacking Grem1, establishment of this E-M feedback signaling loop, distal progression of limb-bud development, and specification of digit identities is disrupted. The BMP antagonist GREM1 defines a regulatory node in this at least in parts self-regulatory limb signaling system as its transcription is positively regulated by BMPs (predominant during initiation) and $\mathrm{SHH}$ (predominant during progression), and inhibited by high FGF levels (predominant during termination of $\mathrm{E}-\mathrm{M}$ feedback signaling).

required to up-regulate AER-FGF signaling and to establish SHH/GREM1/FGF E-M feedback signaling (Fig. 5). In Grem1-deficient mouse limb buds, establishment of E-M feedback signaling is disrupted, which in turn interferes with specification and expansion of the distal limb-bud compartments (zeugopod and autopod) (Khokha et al. 2003; Michos et al. 2004). During initiation of limb-bud development, BMP signaling is, however, required to induce formation of a functional AER and rapidly up-regulates the expression of its own antagonist Grem1 (Fig. 5) (Ahn et al. 2001; Nissim et al. 2006; Bénazet et al. 2009). This results in fast reduction of overall BMP activity, which enables the establishment of the $\mathrm{SHH} /$ GREM1/FGF feedback loop, specification of posterior digits, and distal progression of limbbud development (Bénazet et al. 2009).

Another fascinating aspect of this feedback signaling system is self-termination of the SHH/GREM1/FGF feedback loop due to refractoriness of the expanding population of Shh descendants to activate Grem 1 expression (Scherz et al. 2004; Nissim et al. 2006). In fact, the increasing gap between Shh and Greml expressing cells eventually disrupts $\mathrm{SHH}-$ mediated up-regulation of Grem1 expression, which in turn terminates the SHH/GREM1/ FGF feedback loop (Scherz et al. 2004). Recent genetic analysis of FGF signal reception shows that increasing AER-FGF signaling (see previous discussion) begins to inhibit Grem1 expression in the posterior limb-bud mesenchyme (Verheyden and Sun 2008). Therefore, the GREM1-mediated increase in AER-FGF signaling eventually triggers a FGF/Grem1 inhibitory loop, which self-terminates limb-bud outgrowth and patterning as a consequence of shutting down Grem1 expression (Fig. 5) (Verheyden and Sun 2008). In summary, limb-bud development seems to progress from BMP4-dependent initiation via predominantly SHH-dependent specification and proliferative expansion of the distal autopod primordia to FGF-mediated self-termination (Fig. 5). These interconnected signaling feedback loops define a self-regulatory limb patterning system, mostly because of their differential regulatory impact on Grem1 expression (Bénazet et al., 2009).

\section{CONCLUDING REMARKS}

Despite the fact that the classical studies and models culminated in the identification of the relevant morphogenetic signals and their effectors, they have thus far not provided a comprehensive understanding of vertebrate limb-bud morphogenesis. The molecular analysis in combination with experimental and genetic manipulation has provided important insights into how signals are emitted by ZPA and AER and are received and transduced by responding cells. These studies have now revealed the next level of complexity to be analyzed, namely, 
J.-D. Bénazet and R. Zeller

how information is integrated from different signaling sources and pathways, and memorized such that specification and growth of the whole limb bud occurs in a temporally and spatially coordinated manner (4D patterning). It is likely that early specified, positional information is "checked" continuously and "updated" as the territories are expanded by proliferation to generate the progenitors that form a particular skeletal element. Therefore, identities are likely only "fixed" at rather advanced developmental stages and this determination just precedes the initiation of differentiation. The signaling centers are now well defined both at the cellular and molecular level, which renders the developing limb bud well suited to systems biologytype research approaches. Such genomewide and proteomics-driven functional-genetic approaches in combination with experimental manipulation and the predictive powers of modeling will hopefully provide definitive insights into how signals are sent and received and how information is integrated by cells during specification, proliferation, and determination in time and space.

\section{ACKNOWLEDGMENTS}

We wish to thank A. Roulier for drawing all diagrams, E. Tiecke and M. Osterwalder for providing panels of skeletal stains for Figures 1 and 3, and C. Müller-Thompson for assistance in preparing the text. Our current research is supported by the Swiss National Science Foundation (grant 3100A0-113866), the Novartis Foundation for Medical-Biological Research, and the University of Basel. We sincerely apologize to all colleagues whose work could not be appropriately described and discussed because of the limits and necessary focus of this article.

\section{REFERENCES}

Ahn S, Joyner AL. 2004. Dynamic changes in the response of cells to positive hedgehog signaling during mouse limb patterning. Cell 118: 505-516.

Ahn K, Mishina Y, Hanks MC, Behringer RR, Crenshaw III EB. 2001. BMPR-IA signaling is required for the formation of the apical ectodermal ridge and dorsal-ventral patterning of the limb. Development 128: 4449-4461.

Barna M, Niswander L. 2007. Visualization of cartilage formation: Insight into cellular properties of skeletal progenitors and chondrodysplasia syndromes. Dev Cell 12: 931-941.

Bénazet JD, Bischofberger M, Tiecke E, Gonçalves A, Martin JF, Zuniga A, Naef F, Zeller R. 2009. A self-regulatory system of interlinked signaling feedback loops controls mouse limb patterning. Science 323: 1050-1053.

Boulet AM, Moon AM, Arenkiel BR, Capecchi MR. 2004. The roles of Fgf4 and Fgf8 in limb bud initiation and outgrowth. Dev Biol 273: 361-372.

Capellini TD, Di Giacomo G, Salsi V, Brendolan A, Ferretti E, Srivastava D, Zappavigna V, Selleri L. 2006. Pbx1/Pbx2 requirement for distal limb patterning is mediated by the hierarchical control of Hox gene spatial distribution and Shh expression. Development 133: 2263-2273.

Chen MH, Li YJ, Kawakami T, Xu SM, Chuang PT. 2004. Palmitoylation is required for the production of a soluble multimeric Hedgehog protein complex and long-range signaling in vertebrates. Genes Dev 18: $641-659$.

Chiang C, Litingtung Y, Harris MP, Simandl BK, Li Y, Beachy PA, Fallon JF. 2001. Manifestation of the limb prepattern: Limb development in the absence of Sonic Hedgehog function. Dev Biol 236: 421-435.

Cohn MJ. 2000. Giving limbs a hand. Nature 406: 953-954.

Dahn RD, Fallon JF. 2000. Interdigital regulation of digit identity and homeotic transformation by modulated BMP signaling. Science 289: 438-441.

Dudley AT, Ros MA, Tabin CJ. 2002. A re-examination of proximodistal patterning during vertebrate limb development. Nature 418: 539-544.

Fallon JF, Rowe DA, Frederick JM, Simandl BK. 1983. Studies on epithelial-mesenchymal interactions during limb development. In Epithelial-mesenchymal interactions in development (ed. R.H. Sawyer, J.F. Fallon), 3-25. Praeger, New York.

French V, Bryant PJ, Bryant SV. 1976. Pattern regulation in epimorphic fields. Science 193: 969-981.

Harfe BD, Scherz PJ, Nissim S, Tian H, McMahon AP, Tabin CJ. 2004. Evidence for an expansion-based temporal Shh gradient in specifying vertebrate digit identities. Cell 118: 517-528.

Hornbruch A, Wolpert L. 1991. The spatial and temporal distribution of polarizing activity in the flank of the pre-limb-bud stages in the chick embryo. Development 111: $725-731$.

Khokha MK, Hsu D, Brunet LJ, Dionne MS, Harland RM. 2003. Gremlin is the BMP antagonist required for maintenance of Shh and Fgf signals during limb patterning. Nat Genet 34: 303-307.

Kraus P, Fraidenraich D, Loomis CA. 2001. Some distal limb structures develop in mice lacking Sonic hedgehog signaling. Mech Dev 100: 45-58.

Laufer E, Nelson CE, Johnson RL, Morgan BA, Tabin C. 1994. Sonic hedgehog and $F g f-4$ act through a signaling cascade and feedback loop to integrate growth and patterning of the developing limb bud. Cell 79: 993-1003. 
Lewandoski M, Sun X, Martin GR. 2000. Fgf8 signalling from the AER is essential for normal limb development. Nat Genet 26: 460-463.

Li Y, Zhang H, Litingtung Y, Chiang C. 2006. Cholesterol modification restricts the spread of Shh gradient in the limb bud. Proc Natl Acad Sci 103: 6548-6553.

Litingtung Y, Dahn RD, Li Y, Fallon JF, Chiang C. 2002. Shh and Gli3 are dispensable for limb skeleton formation but regulate digit number and identity. Nature 418: 979-983.

Lu P, Minowada G, Martin GR. 2006. Increasing Fgf4 expression in the mouse limb bud causes polysyndactyly and rescues the skeletal defects that result from loss of Fgf8 function. Development 133: 33-42.

Mann RK, Beachy PA. 2004. Novel lipid modifications of secreted protein signals. Annu Rev Biochem 73: 891-923.

Mariani FV, Ahn CP, Martin GR. 2008. Genetic evidence that FGFs have an instructive role in limb proximal-distal patterning. Nature 453: 401-405.

Martin GR. 1998. The roles of FGFs in the early development of vertebrate limbs. Genes Dev 12: 1571-1586.

Mercader N, Leonardo E, Piedra ME, Martinez AC, Ros MA, Torres M. 2000. Opposing RA and FGF signals control proximodistal vertebrate limb development through regulation of Meis genes. Development 127: 3961-3970.

Michos O, Panman L, Vintersten K, Beier K, Zeller R, Zúniga A. 2004. Gremlin-mediated BMP antagonism induces the epithelial-mesenchymal feedback signaling controlling metanephric kidney and limb organogenesis. Development 131: 3401-3410.

Min H, Danilenko DM, Scully SA, Bonlon B, Ring BD, Tarpley JE, DeRose M, Simonet WS. 1998. Fgf-10 is required for both limb and lung development and exhibits striking functional similarity to Drosophila branchless. Genes Dev 12: 3156-3161.

Moon AM, Capecchi MR. 2000. Fgf8 is required for outgrowth and patterning of the limbs. Nat Genet 26: $455-459$.

Niederreither K, Subbarayan V, Dolle P, Chambon P. 1999. Embryonic retinoic acid synthesis is essential for early mouse post-implantation development. Nat Genet 21: 444-448.

Niederreither K, Vermot J, Schuhbaur B, Chambon P, Dolle P. 2002. Embryonic retinoic acid synthesis is required for forelimb growth and anteroposterior patterning in the mouse. Development 129: 3563-3574.

Nissim S, Hasso SM, Fallon JF, Tabin CJ. 2006. Regulation of Gremlin expression in the posterior limb bud. Dev Biol 299: $12-21$.

Niswander L. 2003. Pattern formation: Old models out on a limb. Nat Rev Genet 4: 133-143.

Niswander L, Tickle C, Vogel A, Booth I, Martin GR. 1993. FGF-4 replaces the apical ectodermal ridge and directs outgrowth and patterning of the limb. Cell 75: 579-587.

Niswander L, Jeffrey S, Martin GR, Tickle C. 1994. A positive feedback loop coordinates growth and patterning in the vertebrate limb. Nature 371: 609-612.

Ohuchi H, Nakagawa T, Yamamoto A, Araga A, Ohata T, Ishimaru Y, Yoshioka H, Kuwana T, Nohno T, Yamasaki $\mathrm{M}$, et al. 1997. The mesenchymal factor, FGF10, initiates and maintains the outgrowth of the chick limb bud through interaction with FGF8, an apical ectodermal factor. Development 124: 2235-2244.

Riddle RD, Johnson RL, Laufer E, Tabin C. 1993. Sonic hedgehog mediates the polarizing activity of the ZPA. Cell 75: 1401-1416.

Riddle RD, Ensini M, Nelson C, Tsuchida T, Jessell TM, Tabin C. 1995. Induction of the LIM homeobox gene Lmxl by WNT7a establishes dorsoventral pattern in the vertebrate limb. Cell 83: 631-640.

Ros MA, Lopez-Martinez A, Simandl BK, Rodriguez C, Izpisua Belmonte JC, Dahn R, Fallon JF. 1996. The limb field mesoderm determines initial limb bud anteroposterior asymmetry and budding independent of sonic hedgehog or apical ectodermal gene expressions. Development 122: 2319-2330.

Sanz-Ezquerro JJ, Tickle C. 2003. Fgf signaling controls the number of phalanges and tip formation in developing digits. Curr Biol 13: 1830-1836.

Saunders JW Jr. 1948. The proximo-distal sequence of origin of limb parts of the chick wing and the role of the ectoderm. J Exp Zool 108: 363-404.

Saunders JW Jr, Gasseling MT. 1968. Ectoderm-mesenchymal interaction in the origins of wing symmetry. In Epithelial-mesenchymal interactions (ed. R. Fleischmajer, R.E. Billingham), 78-97. Williams Wilkins, Baltimore.

Scherz PJ, Harfe BD, McMahon AP, Tabin CJ. 2004. The limb bud Shh-Fgf feedback loop is terminated by expansion of former ZPA cells. Science 305: 396-399.

Scherz PJ, McGlinn E, Nissim S, Tabin CJ. 2007. Extended exposure to Sonic hedgehog is required for patterning the posterior digits of the vertebrate limb. Dev Biol 308: $343-354$.

Sekine K, Ohuchi H, Fujiwara M, Yamasaki M, Yoshizawa T, Sato T, Yagashita N, Matsui D, Koga Y, Itoh N, et al. 1999. Fgf10 is essential for limb and lung formation. Nat Genet 21: $138-141$.

Shubin N, Tabin C, Carroll S. 1997. Fossils, genes and the evolution of animal limbs. Nature 388: 639-647.

Summerbell D, Harvey F. 1983. Vitamin A and the control of pattern in vertebrate limbs. In Limb development and regeneration (ed. Fallon J.F., Caplan A.I.), 109-118. Liss, New York.

Summerbell D, Lewis JH, Wolpert L. 1973. Positional information in chick limb morphogenesis. Nature 244: $492-496$.

Sun X, Mariani FV, Martin GR. 2002. Functions of FGF signalling from the apical ectodermal ridge in limb development. Nature 418: 501-508.

Suzuki T, Hasso SM, Fallon JF. 2008. Unique SMAD1/5/8 activity at the phalanx-forming region determines digit identity. Proc Natl Acad Sci 105: 4185-4190.

Tabin C, Wolpert L. 2007. Rethinking the proximodistal axis of the vertebrate limb in the molecular era. Genes Dev 21: $1433-1442$.

Tanaka M, Cohn MJ, Ashby P, Davey M, Martin P, Tickle C. 2000. Distribution of polarizing activity and potential for limb formation in mouse and chick embryos and possible relationships to polydactyly. Development 127: 4011-4021. 


\section{J.-D. Bénazet and R. Zeller}

Tarchini B, Duboule D, Kmita M. 2006. Regulatory constraints in the evolution of the tetrapod limb anteriorposterior polarity. Nature 443: 985-988.

te Welscher P, Fernandez-Teran M, Ros MA, Zeller R. 2002a. Mutual genetic antagonism involving GLI3 and dHAND prepatterns the vertebrate limb bud mesenchyme prior to SHH signaling. Genes Dev 16: 421-426.

te Welscher P, Zúñiga A, Kuijper S, Drenth T, Goedemans HJ, Meijlink F, Zeller R. 2002b. Progression of vertebrate limb development through $\mathrm{SHH}$-mediated counteraction of GLI3. Science 298: $827-830$.

Thaller C, Eichele G. 1987. Identification and spatial distribution of retinoids in the developing chick limb bud. Nature 327: 625-628.

Theil T, Kaesler S, Grotewold L, Bose J, Ruther U. 1999. Gli genes and limb development. Cell Tissue Res 296: 75-83.

Tickle C. 1981. The number of polarizing region cells required to specify additional digits in the developing chick wing. Nature 289: 295-298.

Tickle C, Summerbell D, Wolpert L. 1975. Positional signalling and specification of digits in chick limb morphogenesis. Nature 254: 199-202.

Tickle C, Alberts BM, Wolpert L, Lee J. 1982. Local application of retinoic acid in the limb bud mimics the action of the polarizing region. Nature 296: 564-565.

Towers M, Mahood R, Yin Y, Tickle C. 2008. Integration of growth and specification in chick wing digit-patterning. Nature 452: 882-886.

Varjosalo M, Taipale J. 2008. Hedgehog: Functions and mechanisms. Genes Dev 22: 2454-2472.

Verheyden JM, Sun X. 2008. An Fgf/Gremlin inhibitory feedback loop triggers termination of limb bud outgrowth. Nature 454: 638-641.

Wanek N, Gardiner DM, Muneoka K, Bryant SV. 1991 Conversion by retinoic acid of anterior cells into ZPA cells in the chick wing bud. Nature 350: 81-83.
Wang B, Fallon JF, Beachy PA. 2000. Hedgehog-regulated processing of Gli3 produces an anterior/posterior repressor gradient in the developing vertebrate limb. Cell 100: 423-434.

Wolpert L. 1969. Positional information and the spatial pattern of cellular differentiation. J Theor Biol 25: 1-47.

Yashiro K, Zhao X, Uehara M, Yamashita K, Nishijima M, Nishino J, Saijoh Y, Sakai Y, Hamada H. 2004. Regulation of retinoic acid distribution is required for proximodistal patterning and outgrowth of the developing mouse limb. Dev Cell 6: 411-422.

Zeller R. 2004. It takes time to make a pinky: Unexpected insights into how SHH patterns vertebrate digits. Sci STKE 2004: pe53.

Zeller R, Duboule D. 1997. Dorso-ventral limb polarity and origin of the ridge: On the fringe of independence? Bioessays 19: $541-546$.

Zeng X, Goetz JA, Suber LM, Scott WJ Jr, Schreiner CM, Robbins DJ. 2001. A freely diffusible form of Sonic hedgehog mediates long-range signalling. Nature 411: 716-720.

Zhu J, Nakamura E, Nguyen MT, Bao X, Akiyama H, Mackem S. 2008. Uncoupling Sonic hedgehog control of pattern and expansion of the developing limb bud. Dev Cell 14: 624-632.

Zúñiga A, Zeller R. 1999. Gli3 (Xt) and formin (ld) participate in the positioning of the polarising region and control of posterior limb-bud identity. Development 126: $13-21$.

Zúñiga A, Haramis AP, McMahon AP, Zeller R. 1999. Signal relay by BMP antagonism controls the SHH/FGF4 feedback loop in vertebrate limb buds. Nature 401: 598-602.

Zwilling E. 1956. Interaction between limb bud ectoderm and mesoderm in the chick embryo. II. Experimental limb manipulation. J Exp Zool 132: 173-188. 


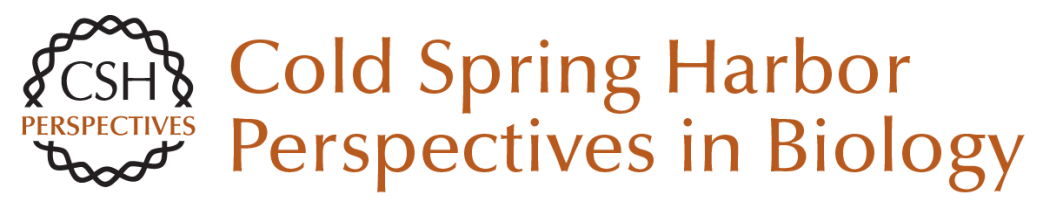

\title{
Vertebrate Limb Development: Moving from Classical Morphogen Gradients to an Integrated 4-Dimensional Patterning System
}

\author{
Jean-Denis Bénazet and Rolf Zeller
}

Cold Spring Harb Perspect Biol 2009; doi: 10.1101/cshperspect.a001339 originally published online July 22, 2009

\section{Subject Collection Generation and Interpretation of Morphogen Gradients}

Regulation of Organ Growth by Morphogen

Gradients

Gerald Schwank and Konrad Basler

Signaling Gradients during Paraxial Mesoderm

Development

Alexander Aulehla and Olivier Pourquié

Morphogen Gradient Formation

Ortrud Wartlick, Anna Kicheva and Marcos

González-Gaitán

Nodal Morphogens

Alexander F. Schier

Gradients and the Specification of Planar Polarity in the Insect Cuticle

David Strutt

Vertebrate Limb Development: Moving from Classical Morphogen Gradients to an Integrated 4-Dimensional Patterning System

Jean-Denis Bénazet and Rolf Zeller

Establishing and Interpreting Graded Sonic Hedgehog Signaling during Vertebrate Neural Tube Patterning: The Role of Negative Feedback Vanessa Ribes and James Briscoe
Gradients in Planarian Regeneration and

Homeostasis

Teresa Adell, Francesc Cebrià and Emili Saló

Shaping Morphogen Gradients by Proteoglycans Dong Yan and Xinhua Lin

Forming Patterns in Development without Morphogen Gradients: Scattered Differentiation and Sorting Out

Robert R. Kay and Christopher R.L. Thompson

Robust Generation and Decoding of Morphogen Gradients

Naama Barkai and Ben-Zion Shilo

Models for the Generation and Interpretation of Gradients

Hans Meinhardt

Graded Dorsal and Differential Gene Regulation in the Drosophila Embryo

Gregory T. Reeves and Angelike Stathopoulos

Chemical Gradients and Chemotropism in Yeast Robert A. Arkowitz

For additional articles in this collection, see http://cshperspectives.cshlp.org/cgi/collection/

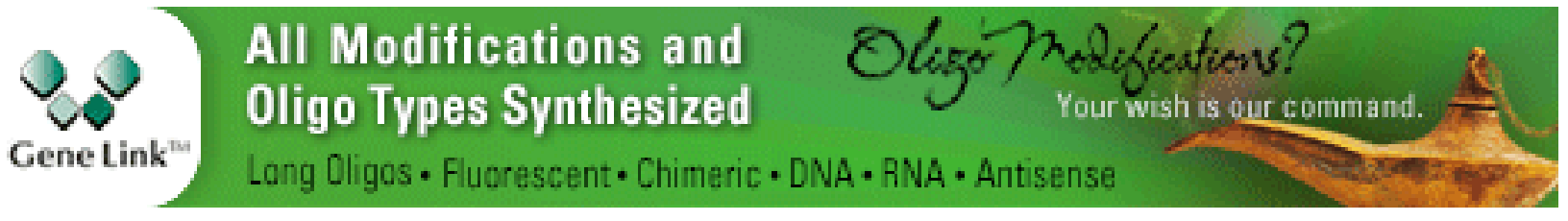


Systems Biology of the Self-regulating

Morphogenetic Gradient of the Xenopus Gastrula Jean-Louis Plouhinec and E. M. De Robertis
Gradients in the Brain: The Control of the Development of Form and Function in the Cerebral Cortex

Stephen N. Sansom and Frederick J. Livesey

For additional articles in this collection, see http://cshperspectives.cshlp.org/cgi/collection/

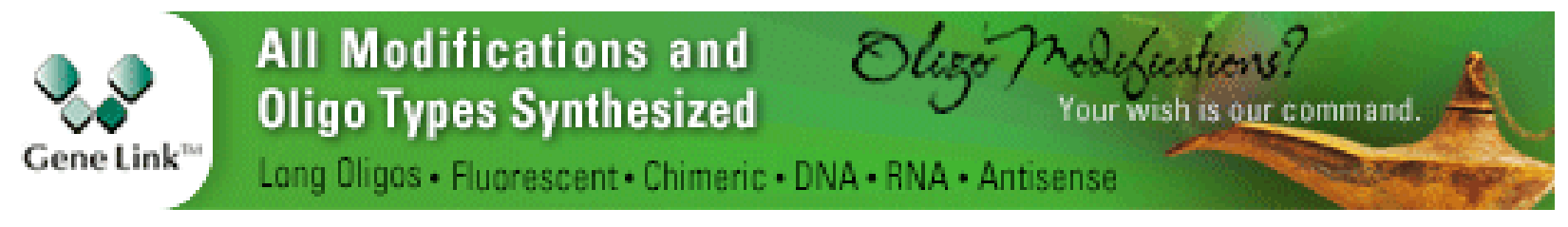

Copyright @ 2009 Cold Spring Harbor Laboratory Press; all rights reserved 